\title{
Les officiers républicains sous l'Empire : entre tradition républicaine, ralliement et tournant libéral
}

\section{Walter Bruyère-Ostells}

\section{(2) OpenEdition}

1 Journals

\section{Édition électronique}

URL : https://journals.openedition.org/ahrf/7533

DOI : 10.4000/ahrf.7533

ISSN : 1952-403X

Éditeur :

Armand Colin, Société des études robespierristes

Édition imprimée

Date de publication : 1 décembre 2006

Pagination : 31-44

ISSN : 0003-4436

\section{Référence électronique}

Walter Bruyère-Ostells, «Les officiers républicains sous l'Empire : entre tradition républicaine, ralliement et tournant libéral », Annales historiques de la Révolution française [En ligne], 346 | Octobre/ Décembre 2006, mis en ligne le 01 décembre 2009, consulté le 22 avril 2022. URL : http:// journals.openedition.org/ahrf/7533; DOI : https://doi.org/10.4000/ahrf.7533

Ce document a été généré automatiquement le 22 avril 2022.

Tous droits réservés 


\title{
Les officiers républicains sous l'Empire : entre tradition républicaine, ralliement et tournant libéral
}

\author{
Walter Bruyère-Ostells
}

1 Pendant la Révolution, l'armée accueille les républicains les plus convaincus. Avec les épurations successives, elle devient un bastion de républicanisme. Il est au moins affiché, comme le montre l'ambition des généraux de servir de «sabre » au coup d'État qui doit jeter à bas le Directoire. Bonaparte assume finalement ce rôle et s'appuie sur une armée dont les convictions sont franchement républicaines. De 1804 à 1815, l'activisme oppositionnel de gauche parait se réduire à des foyers extrêmement limités au sein de la Grande Armée. La partie la plus visible est constituée des complots autour du général Malet. Les historiens en ont longtemps conclu que Napoléon s'est rallié l'armée, que cette dernière est un foyer de bonapartisme. Aujourd'hui fortement nuancée, cette interprétation est très simpliste, même pour les officiers, car elle repose notamment sur une vision nationale. Or, la Grande Armée est de plus en plus européenne au cours de l'Empire : quel sens peut-on donner au bonapartisme pour un Italien ou un Polonais? Est-ce vraiment celui qu'on utilise le plus souvent en France, c'est-à-dire l'attachement à un régime fort dirigé par un Bonaparte? Rien n'est moins sûr.

Quand on se projette vers les années décisives de 1814-1815, ou vers les complots militaires de 1820-1823, on remarque la présence confondue de démocrates et de libéraux aux côtés des "bonapartistes». Ces tendances n'ont donc pas disparu entre 1804 et 1814-1815 ; il paraît sage de replacer la période impériale sur le temps long d'un premier XIX ${ }^{e}$ siècle qui englobe la Révolution. Les mouvances, nées de la politisation intense des armées sous la Révolution, ont simplement été masquées par la légende d'un attachement affectif au " petit caporal ». Cette image a été largement entretenue par le régime. Il n'existe pas forcément chez ces officiers ; il n'induit pas une adhésion 
politique. Au fond, la réflexion sur la culture politique des combattants de la Grande Armée doit être approfondie beaucoup plus qu'elle ne l'a été jusqu'à présent. Tous les outils sont réunis, si on réfléchit à la masse documentaire (archives militaires, mémoires) dont on dispose. Nous ne prétendrons pas ici apporter une réponse mais émettre des hypothèses, lancer des pistes.

3 La culture politique des officiers républicains peut se percevoir selon trois modes d'évolution: persistance d'un sentiment démocrate, conversion à un bonapartisme (qu'il faut redéfinir) de certains officiers et montée du libéralisme, souvent occultée et pourtant flagrante dans les périodes postérieures. Le nombre de députés libéraux de la Restauration issus de la Grande Armée ou le rôle des vétérans en 1830 dans l'instauration du régime de Juillet en sont des preuves manifestes. Pour cela, il faut se pencher sur chacune de ces trois tendances, en commençant par les démocrates, noyau de l'armée semble-t-il en 1804.

Un sentiment républicain éteint ou souterrain ?La République au fond de la conscience

La plupart des officiers se rallient à Bonaparte au début du Consulat. Bonaparte se débarrasse des officiers les plus convaincus en les envoyant à Saint-Domingue; il couvre d'honneurs et de fonctions lointaines les généraux les plus en vue de ce parti (Lannes, Brune et Macdonald nommés ambassadeurs à Lisbonne, Constantinople et Copenhague). Après l'affaire Pichegru et le complot Cadoudal, les chefs républicains sont définitivement écartés : Moreau, condamné à deux ans de prison, est autorisé à passer aux États-Unis ; Delmas et Lecourbe doivent s'exiler et Macdonald est disgracié ${ }^{1}$. La popularité de l'Empereur est à peu près unanime jusqu'à Tilsit, nous dit le colonel Bodinier ${ }^{2}$.

5 Cette unanimité est pourtant trompeuse. Des réseaux d'inspiration démocrate, ou tout au moins des sentiments, subsistent parmi les officiers. Quelques exemples peuvent suffire à éclairer cette assertion. Encore protestataires ou moqueuses sous le Consulat, les figures de proue de la tradition révolutionnaire sont honorées, à l'instar d'Augereau et Lannes. Ils protègent toutefois des officiers démocrates. Cette opinion n'est pas ouvertement exprimée après 1804 ; elle se devine dans les attitudes de certains hommes. Par exemple, Antoine Adolphe Marbot ${ }^{3}$ appartient à la " génération de 1779 " qui a débuté sa carrière sous la Révolution ${ }^{4}$. Il diffère de son frère mémorialiste aux sympathies plus bonapartistes. Toute sa vie, il reste attaché au radicalisme. Encore en 1832, la violence anti-monarchique d'une lettre qu'il adresse à Soult, ministre de la Guerre, en atteste. Ce style est très malhabile, alors qu'il réclame de reprendre du service. Il rappelle par ailleurs « avoir pendant quinze ans employé toutes mes forces à combattre la famille qui nous avait été imposée par l'étranger $»^{5}$. Impliqué dans le complot des «pots de beurre » en $1802^{6}$, Marbot doit son ascension très rapide à la protection de généraux connus pour leur fidélité intransigeante à la Révolution. Bernadotte puis Augereau le prennent tour à tour dans leur état-major de 1800 à 1810 . Ni la Restauration, ni l'Empire ne semblent jamais avoir emporté son adhésion : son opposition à Napoléon Bonaparte cesse après 1802, sans doute plus par peur des représailles que par ralliement au régime. Il n'est pas très zélé au service de l'Empire, comme le prouvent les congés non justifiés qu'il s'accorde. Tous lui sont pardonnés grâce à ses protecteurs, y compris au printemps et à l'été 1812 où il rejoint sans joie son régiment sur la route de la Russie ${ }^{7}$. La fidélité révolutionnaire de cet officier ne s'exprime pas de façon active mais demeure. On peut conclure que Marbot est une conscience radicale sous l'Empire. Il redevient activiste quand les circonstances 
paraissent le permettre : carbonaro sous la Restauration, il se joint au groupe des démocrates et radicaux français transfuges en Espagne en $1823^{8}$. Parmi eux, on compte des étudiants des Amis de la Vérité.

Des velléités d'opposition

6 Autre figure des mouvements nationaux et libéraux après 1815 , Mellinet ${ }^{9}$ franchit un pas plus net dans l'opposition sous l'Empire que Marbot. Engagé volontaire à Nantes (septembre 1792) et placé face aux Vendéens au printemps 1793, il est l'auteur de brochures jacobines dans les années suivantes. Présent aux côtés de Bonaparte le 19 Brumaire $^{10}$, Mellinet bénéficie de la bienveillance du Premier Consul. Le colonel Mellinet est pourtant impliqué dans une affaire de détournement de fonds en Italie à partir de 1806. Malgré la véracité des accusations portées contre lui, après avoir été mis aux arrêts en 1808, il est acquitté en $1811^{11}$. Il est possible que les détournements se soient faits au profit de l'opposition formée autour du général Malet ${ }^{12}$. La mansuétude du tribunal s'expliquerait par les services rendus par Mellinet au moment du coup d'État.

7 Le parcours de cet officier après 1815 confirme son radicalisme politique. Exilé en 1815 pour son ralliement aux Cent-Jours, il est un familier de David et Fouché à Bruxelles. Pendant les années 1815-1830, il annote des œuvres du poète Chénier. Mellinet laisse apparaitre sa culture radicale. Lui-même écrit aussi des odes, dédiées aux grands chefs militaires connus pour leur engagement en faveur de la République, Hoche et Desaix. Il est un des membres fondateurs du club de la Réunion à Bruxelles en 1830 avant de jouer un grand rôle dans la révolution. Impliqué dans le " complot des paniers percés " en Belgique en 1841, il est ensuite régulièrement signalé parmi les convives des banquets démocrates à partir de 1845. Les troupes de volontaires parisiens qu'il avait dirigées en 1830, ont été financées par la très radicale Société des Amis du peuple dont Louis Blanc rappelle «qu'il [lui] fut donné de faire revivre un instant les traditions du club des Jacobins $»^{13}$. Au printemps 1848, Mellinet est condamné à mort pour complot contre la sûreté de l'État. Comme pour de nombreux officiers fidèles au souvenir de la République, l'opposition de Mellinet demeure floue sous l'Empire. Sans doute leur marginalisation au sein de la Grande Armée a-t-elle écrasé toute velléité. Le régime napoléonien a muselé les réseaux oppositionnels.

Il n'est cependant pas impossible que des réseaux démocrates clandestins aient perduré jusqu'en 1806-1808. Le premier complot Malet en mai 1808 espère profiter du départ de Napoléon pour Bayonne ${ }^{14}$. On prévoit d'abolir l'Empire et de rétablir une Assemblée nationale. Depuis les écrits de Charles Nodier ${ }^{15}$, le nom de la mystérieuse société des Philadelphes est évoqué. Peut-être cette société paramaçonnique radicale a-t-elle existé mais, selon les spécialistes, elle n'a pas amené une opposition réellement structurée. Pour semer le doute, il faut toutefois noter que le capitaine piémontais Vittorio Ajmino $^{16}$ est identifié en Italie parmi des conspirateurs radicaux en 1812, au moment du second complot Malet ${ }^{17}$. Cet officier est un futur adhérent des Philadelphes de Buonarroti (sous la Restauration). Cette mouvance comprend peut-être des ramifications insoupçonnées. Même en admettant leur existence, ces réseaux sont marginaux.

Une expression radicale ou démocrate plus aisée dans les périphéries

9 L'historiographie italienne a beaucoup travaillé sur le "jacobinisme» dans la péninsule. À l'instar de Mellinet en poste en Italie, il est sans doute plus facile d'exprimer cette sourde opposition dans les périphéries du Grand Empire. Parmi les 
Napolitains, De Attelis ${ }^{18}$ a rejoint les révolutionnaires de Naples en 1796 et dû s'exiler. Installé à Bologne, il a dirigé un journal radical et s'est signalé comme l'un des fondateurs du « cercle du génie démocratique ». Il est ensuite impliqué en 1798 dans un complot républicain en Toscane ${ }^{19}$. Il sert avec dévouement Joseph puis Joachim sans renier sans doute ses idées. Dans le royaume de Naples, il est possible que les souverains français aient utilisé la franc-maçonnerie militaire pour se rallier les officiers de tradition républicaine. En 1809, De Attelis est ainsi initié à la carboneria. La société secrète va dans un premier temps développer une position anti-muratiste par opposition à la maçonnerie institutionnelle.

Les récriminations anti-impériales ont été plus difficiles à éteindre parmi les Polonais. Vieux combattant, puisqu'il sert déjà en 1792 dans le soulèvement polonais, Grabinski ${ }^{20}$ affiche des opinions très égalitaristes. Son apostrophe à Bonaparte est demeurée célèbre en Italie. Rencontrant le Premier Consul en 1800, il lui lance dans un Français approximatif : "Vous en Italie, moi en Italie; vous en Egypte, moi en Egypte; vous Consul, moi rien ${ }^{21}$. En 1807, il appartient à la légion polonaise de Dabrowski. En raison de la nostalgie républicaine des officiers de cette troupe, Napoléon détache les plus radicaux pour créer la légion de la Vistule. Elle est confiée à Grabinski. La disgrâce du général en chef est de plus en plus visible; il préfère démissionner en $1808^{22}$.

Globalement, les officiers fidèles de pensée à la tradition républicaine ont peu agi contre le régime, à part le cercle très fermé autour du général Malet. Leurs sentiments politiques sont étouffés. La propagande impériale œuvre en ce sens. Aucune structure de sociabilité ne leur permet de faire connaître leurs idées. À Naples, la carboneria naît en partie d'un désir de politisation impossible dans la maçonnerie impériale. Les Français sont plus tenus dans la discipline de fidélité au régime. Déjà sous la Révolution, les radicaux étrangers n'ont pas réussi à élargir leur cercle aux milieux plus populaires. Il est significatif que les radicaux italiens appartiennent essentiellement aux élites, comme le marquis De Attelis ou le prince Pignatelli ${ }^{23}$ à Naples. Sous l'Empire, ces radicaux n'ont comme unique recours que les réseaux clandestins. Très limitée au centre du système napoléonien (Malet), l'opposition souterraine semble avoir plus de marge de manœuvre dans ses périphéries. Elle donne naissance en Italie à la carboneria ${ }^{24}$, promise à un plus grand avenir.

Le ralliement : la Grande Armée vécue comme outil de propagation de l'héritage révolutionnaire?

12 La continuité des guerres révolutionnaires et impériales a sans doute facilité le ralliement de nombreux officiers à l'Empire. C'est pourquoi, entretenue par le régime, une sympathie napoléoniste ${ }^{25}$ se fait jour au sein de la Grande Armée. Elle se verra avec netteté dans les différentes formes d'opposition à la Restauration où l'héritage révolutionnaire soude libéraux, sympathisants bonapartistes et républicains.

Une interprétation difficile du bonapartisme

Napoléon a pu être perçu comme le continuateur de la Révolution, comme l'héritier des Jacobins. Par certains côtés, Napoléon sera toujours le fils couronné de la Révolution, le dictateur de salut public porté sur le trône. D'ailleurs, sans en tirer de conclusion sur sa définition du « parti bonapartiste » en 1815, Frédéric Bluche a noté que $70 \%$ des « oui » motivés au plébiscite de 1815 indiquent leur désaccord sur l'hérédité des pairs. Frédéric Bluche émet l'hypothèse que ces votes seraient républicains ou égalitaristes. Par extension ( $5 \%$ seulement des votes sont motivés), il admet que $60 \%$ des « oui » seraient d'inspiration républicaine ${ }^{26}$. Dans son étude sur les Fédérés qui appuient le régime 
napoléonien des Cent-Jours, Robert $\mathrm{S}$. Alexander arrive à un résultat proche. Il conclut à un attachement à Napoléon perçu comme continuateur de la Révolution mais leurs motivations sont avant tout patriotiques. Selon Robert S. Alexander, ils ne sont pas bonapartistes ${ }^{27}$. Voilà qui met sérieusement en cause l'existence d'un bonapartisme de masse.

Dans cette optique, républicanisme et bonapartisme ne sont pas incompatibles : le vote «bonapartiste » serait un choix par défaut de certains partisans de la République. Plus précisément, l'attachement exprimé à Napoléon ne doit pas être interprété comme une prise de position en faveur de l'Empire. On perçoit d'ailleurs cette proximité en 1815, non chez l'empereur lui-même, mais chez des personnalités qui le poussent à proclamer la patrie en danger et à armer le peuple (Carnot, Lucien Bonaparte). En fait, le bonapartisme relève davantage des sentiments et transcende les courants politiques. Un ralliement plus qu'une adhésion bonapartiste

Le bonapartisme n'existe pas, nous semble-t-il, en tant que force politique identifiée sous l'Empire. On n'observe que des ralliements par sympathie bonapartiste. Ce vocable doit être réservé à l'expression d'un sentiment d'affection envers Napoléon et sa famille. Le général Vaudoncourt ${ }^{28}$ est exemplaire au point que Frédéric Bluche le classe en 1814-1815 comme bonapartiste. Qu'avons-nous pour étayer cette assertion? Il appartient au camp qui penche en faveur de Napoléon en 1815 : il prend part à la défense du territoire national sous les Cent-Jours à la tête de corps francs en Lorraine ${ }^{29}$. Présent en Belgique en 1816, il est associé à une intrigue en faveur de Napoléon II. L'argument paraitt, à première vue, décisif. Cependant, le récit qu'en fait Vaudoncourt éclaire des motivations autres :

«Il ne s'agissait pour moi, ni de la Cour de Napoléon II, ni des dignités qui pouvaient m'y attendre. Je n'ai jamais cherché à ramper dans une Cour, pas même dans celle de mon ancien général en chef [...]. Mon seul but était de sortir ma patrie de l'avilissement où elle était tombée en la délivrant de la domination d'une famille devenue tout à fait antipathique qui ne comprenait ni la nation, ni son siècle et qui ne pouvait que consommer le malheur et la dégradation de la France. Je n'étais pas assez aveugle pour méconnaître la tendance républicaine qui commençait à germer en Europe ; je la partageais assez pour bien la comprendre [...] mais je n'ignorais pas non plus que le nom de république épouvantait encore la masse vulgaire qui s'attache aux noms et leur sacrifie les choses [...]. À ce nom de république, on accolait souvent le sang versé en $1793 »^{30}$.

16 À travers ce passage de ses mémoires (écrits à une époque où rapporter avoir lutté pour Napoléon II n'est plus mal vu), Vaudoncourt affiche sa préférence pour la République. Cette opinion reste beaucoup plus mal perçue par le pouvoir. Son attachement à la République paraît modéré, puisqu'il se réfère aux Lumières et semble condamner de façon implicite les excès de 1793. À son image, beaucoup d'officiers démocrates se rallient et servent avec fidélité le régime qui correspond le mieux à leurs idées. Dans l'esprit de Vaudoncourt, comme de beaucoup de ses contemporains, l'idée républicaine est encore invalidée par l'association avec la Terreur.

17 Le parcours d'un homme comme Cugnet de Montarlot ${ }^{31}$ conforte cette idée. Aucune forme d'opposition, ni même de réprobation, n'est signalée de sa part sous l'Empire. Au contraire, après 1815 , son activisme démocrate est incessant: mêlé à l'intrigue de l'Épingle Noire et à la Conspiration de l'Est, journaliste dans une feuille d'opposition, L'homme gris, membre de sociétés secrètes ${ }^{32}$, il est placé sous surveillance policière ${ }^{33}$. Réfugié en Espagne, ses proclamations éclairent son positionnement politique : 
«La Constitution de 1791, exceptée la puissance royale, est provisoirement proclamée ; elle sera vérifiée de nouveau, et soumise à l'acceptation du peuple qui jurera solennellement de lui rester fidèle. Les deux Chambres de l'État sont dissoutes. Un gouvernement provisoire, composé de 5 membres sera nommé le plus tôt possible et siègera à Lyon jusqu'à ce que Paris ait adopté la Constitution, et arboré le drapeau de l'indépendance [...]. La Garde nationale sera sur le champ réorganisé [...]. M. le général La Fayette est nommé lieutenant des Gardes nationales de France. La composition de l'armée sera la même qu'en 1813. Toutes les troupes seront sur le champ de bataille amalgamées et elles reprendront leur numéro d'ordre : les officiers, les sous-officiers et soldats en activité ou non reprendront leurs rangs $[\ldots] »^{34}$.

Tous les éléments incitent à voir en Cugnet un démocrate : constitution de 1791, rejet de Robespierre, consultation du peuple avant toute mise en place définitive de nouvelles institutions. Le seul nom cité dans les décisions confirme l'orientation modérée, puisqu'il s'agit de La Fayette. Sur le plan militaire, Cugnet de Montarlot inscrit l'Empire dans la continuité, dans l'unité avec la République. L'organisation qu'il préconise est celle de 1813. La légende que la période thermidorienne a forgée du jacobinisme explique sans doute la large adhésion à l'Empire des officiers démocrates. On retrouve le raisonnement plus tard formulé dans la célèbre maxime : «Ah! Que la République était belle sous l'Empire ".

L'influence des combats nationaux dans le ralliement des officiers étrangers

Lydia Scher-Zembitska nuance pourtant l'interprétation des ralliements. Elle note l'exploitation des Polonais par Napoléon sans tenir compte de leurs aspirations nationales avant 1808. Cette réflexion l'amène à considérer que «ceux qui restent à partir de 1808 sont les plus fidèles, ceux qui pensent que Napoléon est bien le seul remède pour restaurer leur pays $»^{35}$. Elle analyse les sentiments politiques des soldats de la Grande Armée de la façon suivante: leur bonapartisme n'est qu'une facette, qu'une arme de la lutte nationale. Leur comportement en 1814-1815 ne devrait, dès lors, plus être perçu comme du bonapartisme mais comme la conviction que seul Napoléon peut garantir l'émergence d'une entité nationale polonaise. On retrouve dans ces ralliés la même préoccupation première de la Nation que chez les radicaux italiens.

Les années 1814-1815 sont très révélatrices. Ces années décisives mettent en lumière de façon plus évidente le choix raisonné des Polonais ou des Italiens (à travers Murat) pour Napoléon face aux monarchies et au risque de Restauration. Surtout parmi les plus jeunes, la "génération des Marie-Louise ", éduquée sous l'Empire et entrée au combat pour la seule période défensive (1813-1815). Dans cette génération, une proportion importante semble affirmer ultérieurement une culture démocrate. Engagé volontaire, Germain Dandelin ${ }^{36}$ est rapidement admis à Polytechnique en 1813. En mars 1814, il combat devant Paris, est blessé et décroche la Légion d'honneur. Revenu en Belgique, il refuse de servir par sympathie pour l'Empire et préfère s'engager au service de la Saxe. Ensuite acteur de l'indépendance belge, il est très remarqué dans des clubs démocrates au point de briser sa carrière alors qu'il est lieutenant-colonel. Il frôle le conseil de guerre et quitte l'armée pour s'adonner à sa passion des... mathématiques ${ }^{37}$. Grandmesnil sert comme chirurgien dans la Garde impériale à partir de 1813. Licencié sous la première Restauration, il reprend du service pour les Cent-Jours. Son attachement à la République se manifeste lors de son implication dans les Chevaliers de la Liberté ${ }^{38}$ et dans la charbonnerie. Son combat anti-monarchique se poursuit sous la Monarchie de Juillet : gérant de journaux démocrates (La Réforme dans les années 1840), il fonde une société républicaine, le "Club des droits de l'homme ${ }^{39}$. 
21 On observe que les hommes le plus souvent qualifiés de bonapartistes ne sont pas de fervents défenseurs de l'Empire. Ce sont soit des «républicains ralliés par sympathie bonapartiste ", soit des "libéraux à sympathie bonapartiste ». En effet, le libéralisme est le dernier courant auquel se convertissent d'anciens officiers républicains.

Du républicanisme au libéralisme

On explique souvent la trahison des maréchaux en 1814 par la lassitude. Il ne s'agit pas ici de remettre en cause ce fait. Il paraît pourtant pertinent de soulever une interrogation de fond. L'échec du ralliement des notables ne doit-il pas être mis en parallèle avec l'échec d'une véritable adhésion bonapartiste chez ces hauts gradés, les hommes les plus proches de Bonaparte, qui ont traversé avec lui l'épopée militaire de la Révolution et de l'Empire. Autant que la lassitude militaire, ne faut-il pas voir dans leur attitude un rejet d'une dictature militaire qui n'a plus de sens dès lors qu'elle ne transmet plus la liberté en Europe et la restreint en France? Ce n'est qu'un postulat mais certains parcours peuvent aller en ce sens. Il semble sage d'établir une distinction entre attachement bonapartiste et adhésion au césarisme moderne ${ }^{40}$ pratiqué par Napoléon Ier : le rejet du second a entraîné l'abandon du premier sentiment.

Des sympathies libérales masquées sous la faveur de Napoléon

Sébastiani peut servir d'archétype de ces militaires. À dix-sept ans en 1789, il quitte les études pour s'engager comme volontaire au Royal-Vintimille. En 1793, devenu souslieutenant, il combat sur son île natale; il est aide de camp du général Rochon et se montre proche du représentant en mission de la Convention Lacombe-Saint Michel. Ses supérieurs notent qu'« il a toujours professé les principes de la Révolution et a travaillé à les propager $\aleph^{41}$. Son positionnement en faveur de la Révolution vaut à sa famille la confiscation de tous ses biens et l'exil en Provence. Rallié à Bonaparte avant Brumaire, protégé du Premier Consul, sa carrière est brillante. En 1810, alors qu'il sert en Espagne, son nom est évoqué dans des intrigues contre Napoléon aux côtés de Soult. Il semble qu'il ait repoussé toutes les sollicitations mais ses rapports avec l'Empereur sont irrémédiablement refroidis. Il figure parmi les ralliés à la première Restauration (décoré de l'ordre de Saint Louis) au point que Napoléon ne lui accorde aucun grand commandement sous les Cent-Jours. Sa connaissance du «complot du Nord $»^{42}$ et le ralliement sur la route de Lille de troupes en fuite vers Gand ne suffisent pas à le faire pardonner.

Il est ensuite un exemple de ces "girouettes » tant raillées : opposition feutrée sous la Restauration comme député libéral, acteur des journées révolutionnaires de juillet 1830, aussitôt rallié à la Résistance. Dans ce dernier changement d'allégeance en 1848, il justifie son action : «Le premier des devoirs de chaque Français est de prodiguer ce qui lui reste de jours pour la défense de sa patrie. J'ai choisi constamment ce principe et je me suis toujours rallié aux hommes qui cherchaient à le faire triompher ${ }^{43}$. Sébastiani explique ses ralliements successifs par le service de la France. Sans doute y voit-on dès l'époque d'autres raisons plus prosaïques. Au moment où il rédige ces lignes, le souci de carrière ne peut pourtant plus jouer pour Sébastiani. Il est maréchal de France, et surtout a déjà 76 ans. Il ne faut pas complètement écarter le désir de cette " génération de 1769 », actrice de la Révolution et de l'Empire, d'imposer les principes pour lesquels elle a combattu à partir de 1789. Parmi ces officiers, les hommes passés de la ferveur enthousiaste pour la République aux grandes heures de la Révolution vers le libéralisme sont nombreux. Ce courant tolère bien des régimes politiques, à condition 
de conserver l'héritage du bouleversement de 1789. Ces libéraux reprennent à leur compte la politique menée par la Révolution jusqu'en 1791-1792.

Un sentiment républicain trop fugace dans une Europe demeurée monarchique

On retrouve ces libéraux dans tous les régimes européens passés sous contrôle français sous la Révolution et l'Empire. La péninsule italienne fait partie de ces zones où la ferveur républicaine a laissé place sur le moyen terme à un monarchisme constitutionnel. Ce mouvement libéral va largement s'exprimer au cours du Risorgimento. De nombreux volontaires ont rejoint les rangs de l'armée républicaine française avec enthousiasme avant d'y faire carrière. Zucchi s'engage comme souslieutenant dans un bataillon de volontaires de Reggio en $1796^{44}$. Le Bolognais Busi est sergent dans l'artillerie cispadane en l'an $\mathrm{VI}^{45}$; Giuseppe Rossaroll entre au service de la république parthénopéenne puis de Bonaparte en $1800^{46} \ldots$

Comme les précédents, le jeune Carascosa ${ }^{47}$ découvre les idéaux républicains au contact des Français. Il a été envoyé en Lombardie par le roi de Naples Ferdinand IV en 1794-1796. Rallié à la Révolution, il sert alors sous Mack et participe à la campagne franco-napolitaine de 1798. Puni par les Bourbons pour avoir rejoint les rangs de la France républicaine, il se rallie à Joseph et le sert fidèlement comme Joachim ensuite. Sous la Restauration, il n'a de cesse de plaider pour une monarchie constitutionnelle libérale ${ }^{48}$. Les Bourbons ne lui pardonnent pas son ralliement à la révolution de 1820-1821; les radicaux et même les démocrates (à commencer par le général Pepe) et les carbonari lui en veulent de son modérantisme.

Très tôt, des radicaux notoires basculent vers le libéralisme, à l'instar du général Bernadotte. Quelle étonnante trajectoire que celle du petit sergent de l'An II, qui se fait tatouer sur le bras "Mort aux tyrans!", devenu le prince Charles Jean, héritier de Suède ! Au sortir de la Révolution, la phraséologie de Bernadotte est encore nettement républicaine, comme le montrent ses proclamations: «Militaires républicains, la bannière de nos ennemis est: Courage, Trahison, Despotisme! La nôtre est : Loyauté, Courage, Liberté ! »; ou encore : «Les soldats du prétendu Louis XVIII aussi lâches que le maître qu'ils servent, ont osé troubler votre sommeil. Réveillés par les accents plaintifs de vos femmes et de vos enfants, vous avez saisi les armes terribles aux ennemis de votre repos et de votre liberté $»^{49}$. Au début du Consulat, ses vues n'ont guère évolué puisqu'on peut raisonnablement voir sa main derrière les complots « des libelles » ou "des pots à beurre ». Le ton est très différent quelques années plus tard lorsqu'il décrit à Napoléon « les plaies d'une révolution dont il ne reste à la France que les souvenirs de la gloire militaire et des malheurs réels dans son intérieur $\rrbracket^{50}$. Après son accession au trône de Suède, le ton est également parfaitement conforme aux tournures conventionnelles de la monarchie : pas la moindre inflexion ne marque les anciens sentiments de Bernadotte.

On pourrait croire que le basculement de Bernadotte n'est que le résultat de son pragmatisme face aux événements ou celui de ses ambitions. Rien n'est moins sûr. Dans son ombre, d'autres parcours sont parallèles à celui du maréchal devenu roi, à commencer par celui de son ami Gérard. Depuis 1793, il appartient aux proches de Bernadotte. En 1802, comme Marbot, il trempe dans les intrigues contre le Premier Consul (affaire des libelles). Il reste proche de Bernadotte pendant toute la période impériale. En avril 1814 encore, il quitte son poste pour aller voir le roi de Suède. En service dans la Grande Armée des Cent-Jours, placé en demi-solde par la seconde Restauration, Gérard demande l'autorisation de voyager vers la Suède. Engagé 
volontaire dès 1791, lieutenant dans l'état-major de Bernadotte en 1793, il suit ensuite le parcours de son général en chef et évolue, comme lui, vers un monarchisme parlementaire. Sous la première Restauration, la police royale s'inquiète de sa correspondance avec Davout, il doit aussi justifier une entrevue chez lui avec La Bédoyère et de nombreux autres officiers ${ }^{51}$. Il aurait pris part aux conciliabules $d u$ "Complot du Nord $»^{52}$. Non bonapartiste, Gérard prend alors des contacts avec les activistes de ce courant : son libéralisme l'emporte sur des convictions dynastiques. Dès lors, son parcours sous la Restauration et pendant la Révolution de 1830 se comprend mieux. Revenu en France en 1817, il siège comme député libéral à partir de 1822 et appuie l'accession au trône de Louis-Philippe au cours des journées révolutionnaires de juillet 1830.

L'attachement à la République s'efface apparemment parmi les officiers sous l'Empire. Une partie de ces hommes conservent pourtant leurs opinions radicales ou démocrates. Ils les taisent tous et certains tentent de recréer des réseaux souterrains. La marge de manœuvre permise par l'éloignement de Paris permet d'avancer l'hypothèse que l'activisme démocrate ou radical est plus grand dans les périphéries de l'Empire qu'en France. La persistance d'idéaux démocrates sous l'Empire explique aussi le regain de cette tendance politique au sein de la "génération des Marie-Louise", ou de la "génération de 1820 " définie par l'historien américain Alan B. Spitzer ${ }^{53}$. On voit resurgir cette famille politique parmi les officiers, alors que le contrôle impérial se relâche. La plupart des officiers de la République se rallient à l'Empire et même adoptent des idées libérales. Cela ne signifie pas qu'ils ne sont pas les récepteurs d'une partie de l'héritage de la République. Beaucoup combattent pour la liberté, l'égalité (notamment dans l'accès au grade d'officier) et la fraternité (en voulant apporter les bienfaits de la Révolution à l'Europe). Chimère peut-être mais qui explique en partie la fidélité globale jusqu'en 1814. Certains vont continuer ce combat au service des mouvements nationaux et libéraux. Les Italiens le font dans la péninsule, les Polonais ou les Belges sur leur territoire occupé, mais d'autres hommes rejoignent la Grèce, l'Amérique... Sous l'Empire, il n'y a pas, ou presque pas, d'officiers bonapartistes au sens politique du terme. Au-delà des sympathies plus ou moins grandes pour l'Empereur, les officiers napoléoniens se rangent en deux grandes catégories: les démocrates et les monarchistes constitutionnels. Ces derniers ont, ou non, une sympathie dynastique après 1815 .

\section{NOTES}

1.Claude CROUBOIs (dir.), L'officier des origines à nos jours, St-Jean-d'Angély, Éditions Bordessoules, 1987, 429 p. Le chapitre consacré à l'Empire est rédigé par le commandant Bodinier.

2.Idem, $\mathrm{p} 103$.

3.Antoine Adolphe Marbot (1781-1844). Il s'engage en 1798.

4.Dans notre travail de thèse (Walter BRUYÈRE-OSTELLS, Les officiers de la Grande Armée dans les mouvements nationaux et libéraux 1815-1833, sous la direction de Jacques-Olivier 
BouDON, Paris-IV, 2005, 683 p.), il nous a semblé important de distinguer plusieurs générations d'officiers au sein de la Grande Armée (d'autres historiens ont déjà fait le même constat, parmi lesquels Natalie Petiteau). La " génération de 1779 » comprend des hommes engagés dès la Révolution tout en n'ayant pas atteint l'âge de dix-huit ans en 1789. L'ambiance révolutionnaire a marqué leur esprit d'adolescent ; pour certains, leur destin personnel et familial a été bouleversé par le cours de la Révolution.

5.Lettre adressée en 1832 à Soult, ministre de la Guerre (Service historique de la Défense, 8 Yf 2 844).

6.Le complot des « pots de beurre » ou " des libelles », qui avait pour but de renverser le premier consul, n'avait connu qu'un début d'exécution : l'envoi de libelles républicains de Bretagne dans les pots de beurre. Bernadotte est sans doute à l'origine du projet mais il sera couvert par le général Simon, seul condamné (réintégré mais exilé en Champagne par Napoléon en 1804).

7.Service historique de la Défense, 8 Yf 2844.

8.AN, F7 6682.

9.Anne François Mellinet, colonel (né à Corbeil en 1768, mort en 1852).

10.Service historique de la Défense, 16 Yd 200.

11.Service historique de la Défense, 16 Yd 200.

12.Louis LECONTE, Le général A.F. Mellinet et sa brigade « la victorieuse », Bruxelles, Éditions l'Avenir, 1932, $130 \mathrm{p}$.

13. Louis Blanc, Histoire de dix ans, tome 2, p 326 (il confirme l'envoi de volontaires en Belgique).

14.On n'entrera pas dans les détails des complots Malet, relativement connus.

15. Charles NODIER (1780-1844), écrivain français, se présente comme le fondateur des Philadelphes en 1797, dans son Histoire des sociétés secrètes de l'armée et des conspirations contre Bonaparte, Paris, Gide, 1815.

16.Né en 1781 à Borgomasino (province d'Ivrea), Ajmino avait participé aux troubles révolutionnaires en Piémont en 1797-1798.

17.Josiane Bourguet-Rouveyre, Les Piémontais face à l'annexion française 1798-1814, thèse de doctorat, Université Paris-I, 1992, 575 p.

18.De Attelis (Orazio), marquis, né en 1774 à San Angelo Limosano (Pouilles). 19. Alberto GIL NovalÈs, Diccionario biografico del trienio liberal, Madrid, Ed. El Museo universal, 1991.

20.Né en Pologne en 1767 dans une famille de moyenne noblesse, Grabinski sert dans les rangs français de 1797 à 1808.

21.Anecdote rapportée par Krzysztof Zaboklicki, « Da ufficiale napoleonico a protagonista del Risorgimento bolognese », Rassegna storica del Risorgimento, Roma, Istituto per la storia del Risorgimento, 1993, anno LXXX-IV, p 478 (traduction). 22.Idem, p. 482-83.

23.Francesco Pignatelli, prince de Strongoli (né en 1775), général dans l'armée du royaume de Naples.

24.Sans lier la naissance de cette société secrète à la seule opposition démocrate. 25.On entendra par ce terme une admiration et un attachement affectif à l'homme Napoléon. Elle ne sera jamais unanime et n'empêche pas les récriminations dans les rangs.

26.Frédéric BLUCHE, Le plébiscite des Cent-Jours (avril-mai 1815), Genève, Droz, 1974, 149 p.

27. Robert S. ALEXANDER, Bonapartism and Revolutionary Tradition : the Fédérés of 1815, Cambridge, Cambridge University Press, 1991, 314 p. 
28.Frédéric Guillaume de Vaudoncourt (1772-1845), général et baron du royaume d'Italie à partir de 1809 .

29.Service historique de la Défense, 7 Yd 1822. Cet événement semble le principal argument avancé par Frédéric Bluche pour attester de son bonapartisme, compte tenu de la présence d'autres supposés chauds partisans de l'Empire à ses côtés (le colonel Viriot, le capitaine Brice).

30.Guillaume de VAudoncourt, Quinze ans d'un proscrit, Paris, Duffrey, 1835, 4 volumes. 31.Claude François Cugnet de Montarlot (1778-1824), engagé dans l'infanterie en 1797, est commissaire de guerre à partir de 1807.

32.BNF, fichier Bossu et F-M 3, 599.

33.Archives de la préfecture de police, Ea/70, 16 et AN, F7 6688 .

34.AN, F7 6649.

35.Lydia SCHER-ZEMBITSKA, Les Polonais au service de la France de 1732 à 1832, thèse microfilmée sous la direction d'André CORvISIER, Paris-IV, 1993, 2 volumes. Les principaux développements sont repris dans son ouvrage, L'aigle et le phénix, un siècle de relations franco-polonaises 1732-1832, Paris, CNRS Éditions, 2001, 469 p.

36.Germain Dandelin, né en 1794, est sous-lieutenant en 1815.

37.Musée royal de l'armée (KLM-MRA), officier n 145

38.Il en est l'un des fondateurs (BNF, fichier Bossu)

39.Service historique de la Défense, 3 Yg 446.

40.Cette notion paraît plus correcte pour décrire la pratique politique napoléonienne et ses partisans que le terme très généraliste de bonapartisme. Elle fait désormais l'unanimité chez les politologues.

41.Service historique de la Défense, $6 \mathrm{Yd} 40$.

42.Walter BRUYÈRE-OSTELLS, «L'armée, foyer de bonapartisme : l'exemple du complot du Nord en 1815 ", dans Armée, guerre et société à l'époque napoléonienne, Paris, SPM, collection de l'Institut Napoléon, 2004, p 81-87.

43.Lettre de Sébastiani au ministre de la Guerre du gouvernement provisoire le 27 février 1848 (Service historique de la Défense, 6 Yd 40).

44.Carlo Zucchi (1777-1863) fait carrière au service de Napoléon et devient baron d'Empire. Ses sentiments libéraux se manifestent en 1830-1831 avec son ralliement à François IV de Modène (complot Ciro-Menotti) puis au cours de la révolution d'EmilieRomagne (Archivio di Stato Milano, Matricole degli ufficiali, 27).

45.Clemente Busi, né en 1776, sert fidèlement dans l'armée impériale. Passé au service du Pape, il rallie la révolution de 1831 et se montre comme un des officiers proches d'Armandi. Archivio di Stato Milano, Matricole degli ufficiali, 76 ; Service historique de la Défense, XL 66.

46.Giuseppe Rossaroll (1775-1825), comme le rappelle la police à son propos (Archivio di Stato Napoli, Ministero di Polizia, 4 605).

47. Michele Carascosa (1774-1852).

48.Ses mémoires sont très explicites sur ces opinions politiques, notamment lors de son affrontement avec Pepe au cours de la révolution de 1820-1821. En France, il n'y a pas d'exemplaire de ces Mémoires historiques, politiques et militaires sur la révolution de Naples en 1820 et 1821 et sur les causes qui l'ont amenées, Londres, Treuttel-Würtz-Richter, 1823, XII-564 p. Voir : Archivio di Stato Napoli, côte Archivio Borbone, 2528.

49.Extraits des proclamations des 8 et 15 Fructidor an VII ( 25 août et 1er septembre 1799).

50.Lettre du 20 mars 1813. 
51.Lettre de Gérard au ministre de la Police générale du 15 novembre 1815 à propos de la première Restauration (Service historique de la Défense, $6 \mathrm{Yd} 36$ ).

52.La Bédoyère est impliqué dans les préparatifs et Davout devait en prendre la tête avant de se désister (Walter Bruyère-Ostells, « L'exemple du complot du Nord en 1815 », op. cit.).

53.Alan B. SPITZER, The French Generation of 1820, Princeton, Princeton University Press, 1987, XVI-335 p.

\section{RÉSUMÉS}

Le ralliement des officiers à l'Empire est trompeur : il masque de nombreuses consciences restées démocrates ou radicales. Dans les périphéries du Grand Empire (Italie), on relève des velléités d'opposition, voire peut-être l'existence de réseaux clandestins (Philadelphes). Cependant, aucune structure de sociabilité ne permet l'éclosion d'un mouvement oppositionnel. Malgré les ralliements, parler $\mathrm{du}$ bonapartisme des officiers n'a guère de sens. En fait, il n'est pas un sentiment politique. Le bonapartisme doit davantage qualifier un sentiment de sympathie, d'attachement des officiers envers Napoléon qu'une adhésion au régime mis en place (césarisme moderne). La plupart des officiers fidèles sont cependant des libéraux, ce qui explique leur carrière de " girouettes » à partir de 1814. L'Empire est un moment fort de conversion d'officiers d'horizons divers (jusqu'aux anciens jacobins) au libéralisme politique.

Republican Officiers under the Empire : between the Republican Tradition, rallying of Military Officiers and the Liberal Turn. The rallying of officers to the Empire is misleading : it conceals the fact that many among them remained democrats and radicals in their private convictions. On the margins of the Grand Empire (Italy), latent opposition was present, perhaps even clandestine networks (Philadelphes). But no structure of sociability permitted the birth of an oppositional movement. Despite the rallying of officers, to speak of a Bonapartism among the officers is without foundation. Far from being a political sentiment, Bonapartism was rather a feeling of sympathy, more an attachment of officers towards Napoleon than an adherence to the established regime (modern cesarism). Most of the faithful officers are, however, liberals, which explains their career as political turncoats beginning in 1814. The Empire was a time that saw the conversion of military officers of divers political horizons (even including former Jacobins) to political liberalism.

\section{INDEX}

Mots-clés : culture politique, libéralisme, officiers, césarisme, bonapartisme

\section{AUTEUR}

\section{WALTER BRUYÈRE-OSTELLS}

11 av. du Pont Juvenal, 34000 Montpellier, walter.bo@caramail.com 